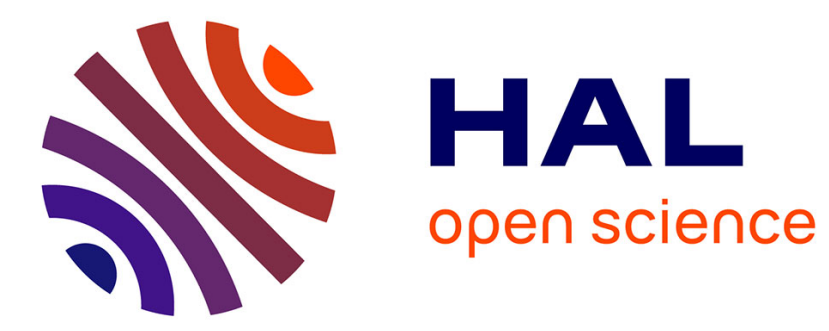

\title{
Observation of a Complex Solar Wind Reconnection Exhaust from Spacecraft Separated by over 1800 R E
}

B. Lavraud, J. T. Gosling, A. P. Rouillard, A. Fedorov, A. Opitz, J.-A. Sauvaud, C. Foullon, I. Dandouras, Vincent Génot, C. Jacquey, et al.

\section{- To cite this version:}

B. Lavraud, J. T. Gosling, A. P. Rouillard, A. Fedorov, A. Opitz, et al.. Observation of a Complex Solar Wind Reconnection Exhaust from Spacecraft Separated by over 1800 R E. 2009. hal-00377237

\section{HAL Id: hal-00377237 \\ https://hal.science/hal-00377237}

Preprint submitted on 21 Apr 2009

HAL is a multi-disciplinary open access archive for the deposit and dissemination of scientific research documents, whether they are published or not. The documents may come from teaching and research institutions in France or abroad, or from public or private research centers.
L'archive ouverte pluridisciplinaire HAL, est destinée au dépôt et à la diffusion de documents scientifiques de niveau recherche, publiés ou non, émanant des établissements d'enseignement et de recherche français ou étrangers, des laboratoires publics ou privés. 


\title{
Observation of a Complex Solar Wind Reconnection Exhaust from Spacecraft Separated by over $1800 R_{E}$
}

\author{
B. Lavraud, ${ }^{1,2}$ J. T. Gosling, ${ }^{3}$ A. P. Rouillard, ${ }^{4}$ A. Fedorov, ${ }^{1,2}$ A. Opitz, ${ }^{1,2}$ J.-A. \\ Sauvaud, ${ }^{1,2}$ C. Foullon, ${ }^{5}$ I. Dandouras, ${ }^{1,2}$ V. Génot, ${ }^{1,2}$ C. Jacquey,,${ }^{1,2}$ P. \\ Louarn, ${ }^{1,2}$ C. Mazelle, ${ }^{1,2}$ E. Penou, ${ }^{1,2}$ T. D. Phan, ${ }^{6}$ D. E. Larson, ${ }^{6}$ J. G. \\ Luhmann, ${ }^{6}$ P. Schroeder, ${ }^{6}$ R. M. Skoug, ${ }^{7}$ J. T. Steinberg, ${ }^{7}$ and C. T. Russell ${ }^{8}$
}

\footnotetext{
${ }^{1}$ Centre d'Etude Spatiale des Rayonnements, Université de Toulouse (UPS), 9 Avenue du Colonel Roche, 31028 Toulouse Cedex 4, France

${ }^{2}$ Centre National de la Recherche Scientifique, UMR 5187, Toulouse, France

${ }^{3}$ Laboratory for Atmospheric and Space Physics, University of Colorado, Boulder, Colorado, USA

${ }^{4}$ Space Environment Physics Group, School of Physics and Astronomy, University of Southampton, UK

${ }^{5}$ Mullard Space Science Laboratory, University College London, Holmbury St Mary, Dorking, Surrey, UK

${ }^{6}$ Space Sciences Laboratory, University of California, Berkeley, USA

${ }^{7}$ Space Science and Applications, Los Alamos National Laboratory, Los Alamos, New Mexico, USA

${ }^{8}$ Institute of Geophysics and Planetary Physics, University of California, Los Angeles, USA
}

\begin{abstract}
We analyze Wind, ACE and STEREO (A and B) plasma and magnetic field data in the vicinity of the heliospheric current sheet (HCS) crossed by all spacecraft between 22:15 UT on March 31 and 01:25 UT on April 1, 2007; corresponding to its observation at ST-A and ST-B which were separated by over $1800 R_{E}$ (or over $1200 R_{E}$ across the Sun-Earth line). Although only Wind and ACE provided good ion flow data in accord with a solar wind magnetic reconnection exhaust at the HCS, the magnetic field bifurcation typical of such exhausts was clearly observed at all spacecraft. They also all observed unambiguous strahl mixing within the exhaust, consistent with the sunward flow deflection observed at Wind and ACE and thus with the formation of closed magnetic field lines within the exhaust with both ends attached to the Sun. The strong dawnward flow deflection in the exhaust is consistent with the exhaust and $\mathrm{X}$-line orientations obtained from minimum variance analysis at each spacecraft so that the X-line is almost along the GSE Z-axis and duskward of all the spacecraft. The observation of strahl mixing in extended and intermittent layers outside the exhaust by ST-A and B is consistent with the formation of electron separatrix layers surrounding the exhaust. This event also provides further evidence that balanced parallel and anti-parallel suprathermal electron fluxes are not a necessary condition for identification of closed field lines in the solar wind. In the present case the origin of the imbalance simply is the mixing of strahls of substantially different strengths from a different solar source each side of the HCS. The inferred exhaust orientations and distances of each spacecraft relative to the $\mathrm{X}$-line show that the exhaust was likely non-planar, following the Parker spiral orientation. Finally, the separatrix layers and exhausts properties at each spacecraft suggest that the magnetic reconnection X-line location and/or reconnection rate were variable in both space and time at such large scales.
\end{abstract}

\section{Introduction}

The process of magnetic reconnection converts magnetic energy into particle kinetic and thermal energy. It occurs ubiquitously from laboratory plasmas to stars. It has in particular been largely studied in Earth's magnetosphere (Dungey, 1961). In situ observations at Earth's magnetopause have shown that magnetic reconnection is characterized by bulk acceleration and heating of both ions and electrons (e.g., Paschmann et al., 1979; 1993; Sonnerup et al., 1981; Gosling et al., 1986). At the magnetopause, the high energy tails of the ion and electron distribution functions are capable of producing separatrix layers that are observable to some distance away from the reconnecting boundary, owing to their large parallel velocities together with velocity filter effects 
associated with plasma convection (Gosling et al., 1990). This property permits confirmation of the local openness of the magnetopause, since particles with the characteristics from one side of the open magnetopause can be observed on the other side (Fuselier, Anderson, and Onsager, 1995; 1997). This further allows inference of the global magnetic topology and solar wind plasma entry mechanism into the magnetosphere (e.g., Onsager et al., 2001; Lavraud et al., 2005; 2006).

Recently, magnetic reconnection has been shown to occur frequently in the solar wind (Gosling et al., 2005a; 2007a; Phan et al., 2006; Davis et al., 2006; Huttunen, Bale, and Salem, 2008). Gosling et al. (2005b; 2007a) showed that magnetic reconnection at the heliospheric current sheet (HCS) in the solar wind leads to the observation of apparent magnetic disconnection from the Sun if the spacecraft is anti-sunward of the X-line along the reconnected magnetic field lines. Conversely, Gosling et al. (2006) showed that magnetic reconnection at the HCS concomitantly leads to newly closed magnetic field lines with both ends attached to the Sun, as observed if the spacecraft is sunward of the X-line. Magnetic disconnection from the Sun and the formation of closed field lines sunward of the reconnection site were demonstrated using measurements of solar wind suprathermal electrons. The former case leads to electron heat-flux (strahl) drop-outs in the exhaust along with interpenetrating back-streaming halo electrons, while the latter case leads to bidirectional suprathermal electron strahls as illustrated in Figure 1 (cf. also Figure 5 of Gosling et al. (2006)).

Magnetic reconnection in the solar wind creates a bifurcated current sheet; these two edge boundaries resemble rotational discontinuities (Gosling et al., 2005a). This configuration is depicted in Figure 1. The exhaust region contains plasma accelerated into it as the field line kinks produced by reconnection propagate into the upstream solar wind. However, in a fashion similar to what is observed at Earth's magnetopause, suprathermal electrons are not confined to the exhaust region, but rather may leak out and form separatrix layers on either side of the exhaust (Gosling et al., 2005b; 2006; Huttunen, Bale, and Salem, 2008). As the reconnection exhaust propagates away from the X-line, its spatial width is expected to increase. In a special case where magnetic reconnection likely may have been initiated very close to the Sun, Gosling et al. (2007a) found exhausts lasting several hours at several spacecraft.

Unlike at Earth's magnetopause where magnetic reconnection can often be patchy in both space and time (Russell and Elphic, 1978; 1979) as well as quasi-stationary (e.g., Paschmann et al., 1979; Sonnerup et al., 1981), magnetic reconnection in the solar wind appears to be quasi-stationary along extended X-lines (e.g., Phan et al. 2006; Gosling et al. 2007a; 2007b).

\section{Instrumentation}

The Solar Terrestrial Relations Observatory (STEREO; Kaiser et al., 2008) consists of two spacecraft that slowly drift ahead (referred to as ST-A) and behind (referred to as ST-B) the Earth on similar orbits. We use data from the Solar Wind Electron Analyzers (SWEA) (Sauvaud et al., 2008) and MAGnetometers (MAG) (Acuña et al., 2008) from the In-situ Measurements of PArticles and CME Transients (IMPACT) instrument suite (Luhmann et al., 2008). Identical instruments are present on each of the two STEREO spacecraft. The two spacecraft are nonspinning due to the solar imaging requirements of the mission. The SWEA instruments are top-hat electrostatic analyzers with an additional deflection system (from setting a finite potential on two curved plates at its entrance) that allows measurement of incoming particles to angles of $\pm 65^{\circ}$ from the regular top-hat entrance (that looks in the plane perpendicular to the Sun-spacecraft direction), in the energy range from about 0 to $2 \mathrm{keV}$. The SWEA instruments are thus able to measure most of the electron distribution function ( $\sim 80 \%$ of the $4 \pi$ solid angles) apart from two cones in the solar and anti-solar directions. 
We use high-resolution plasma (Lin et al., 1995) and magnetic field (Lepping et al., 1995) data from the Wind spacecraft for the identification of the solar wind reconnection exhaust at the HCS on March 31, 2007. The exhaust is also observed at the Advanced Composition Explorer (ACE) from plasma (McComas et al., 1998) and magnetic field (Smith et al., 1998) data, but at both STEREO spacecraft is only clearly determined from magnetic field and suprathermal electron data. All data used are in Geocentric Solar Ecliptic (GSE) coordinates.

Suprathermal electron data from STEREO come from $2 \mathrm{~s}$ three-dimensional (3D) distributions obtained every 30 s. Those from Wind (ACE) are from $3 \mathrm{~s}$ obtained every $98 \mathrm{~s}$ (64 s). All suprathermal electron pitch angle distributions (PAD) are calculated in the solar wind flow frame. Note that SWEA data are not yet calibrated in an absolute fashion, but the PAD shapes are correct owing to implementation of appropriate inter-anode and interdeflection calibrations.

\section{Observations}

Around the end of March 2007 a fast solar wind stream was catching up with slow solar wind in the vicinity of Earth at 1 AU. The locations of the ACE, Wind, ST-A and B spacecraft (Figure 2) allowed the sampling of the resulting corotating interaction region with a large-scale perspective. After more than two days of slow solar wind $(<400 \mathrm{~km} / \mathrm{s})$, all spacecraft encountered the HCS, followed by the stream interfaces (SI; 2:37 and 4:00 UT on April 1 respectively at ST-A and B) a few hours later. A couple days after the SI passage, all spacecraft were eventually in the pristine high speed stream with speeds reaching up to $\sim 700 \mathrm{~km} / \mathrm{s}$.

Figure 2(a) shows the positions of the spacecraft projected into the X-Y $Y_{\text {GSE }}$ plane at 22:30 UT on March 312007 , together with the orientation of the intersection with that plane of the exhaust produced by magnetic reconnection at the HCS. The HCS was observed almost simultaneously at ACE and ST-A at 22:30 UT on March 31, 2007. This timing provides the orientation demarked by the solid red line in Figure 2(a). The dashed lines show the exhaust intersection with the $\mathrm{X}-\mathrm{Y}_{\mathrm{GSE}}$ plane as deduced from Minimum Variance Analysis (MVA; Sonnerup and Scheible (1998)) at each spacecraft (further discussed later). Figure 2(b) shows the location of the spacecraft in the $\mathrm{Y}-\mathrm{Z}_{\mathrm{GSE}}$ plane, with ST-B somewhat southward of the other spacecraft. The X-line orientation projected into that plane is drawn in Figure 2(b) from the interpretation of the MVA results at each spacecraft, as detailed later. As can be seen in Figure 2(a), the two STEREO spacecraft were separated by over $1800 R_{E}$ and $\sim 1200 R_{E}$ across the Sun-Earth line.

Figure 3 shows high resolution data from Wind (see captions for details). $\mathrm{B}_{\mathrm{X}}$ and $\mathrm{B}_{\mathrm{Y}}$ (panel (e)) change sign as expected across the HCS observed at 22:32 UT. The suprathermal electron spectrogram (panel (a)) shows a $0^{\circ}$ strahl, indicating electron flow parallel to the magnetic field, before $\sim 22: 32$ UT and a $180^{\circ}$ strahl after, consistent with a crossing of the HCS. The total magnetic shear across the HCS is about $90^{\circ}$. The magnetic field change at the HCS occurred in two distinct steps, indicative of a bifurcated current sheet. These field changes bounded a region of accelerated plasma flow lasting about $1 \mathrm{~min} 20 \mathrm{~s}$ (marked by vertical black lines). The bulk plasma flow change had both a sunward and a dawnward deflection (panel (d)). The spacecraft observed correlated changes in $\boldsymbol{V}$ and $\boldsymbol{B}$ components as it entered the exhaust and anti-correlated changes in $\boldsymbol{V}$ and $\boldsymbol{B}$ components as it exited the exhaust. This is expected since Alfvénic disturbances propagating parallel (anti-parallel) to $\boldsymbol{B}$ produce anti-correlated (correlated) changes in $\boldsymbol{V}$ and $\boldsymbol{B}$, respectively. Both ion density and temperature (panels (b) and (c)) were enhanced within the exhaust and the field magnitude was depressed (panel (f)), as is often the case within reconnection exhausts in the solar wind (Gosling et al., 2005a).

MVA analysis across the exhaust allows us to infer the intersection of the exhaust plane (from knowledge of the direction normal to the exhaust; the direction of minimum variance $\mathrm{N}$ ) with the $\mathrm{X}-\mathrm{Y}_{\mathrm{GSE}}$ plane, as depicted in Figure 
2(a) with a red dashed line for Wind. MVA also informs us on the orientation of the magnetic reconnection X-line. For an ideal magnetic reconnection exhaust with a constant guide field across it, the X-line orientation is given by the MVA intermediate eigenvector direction (M; cf. Phan et al. (2006) and Gosling et al. (2007b)). The projection of the $\mathrm{X}$-line orientation into the $\mathrm{Y}-\mathrm{Z}_{\mathrm{GSE}}$ plane is shown in Figure 2(b) with a red dashed line. The $\mathrm{L}$ direction is that of maximum magnetic field variance and corresponds to the direction of exhaust propagation (cf. Figure 1). At Wind, the normal direction to the exhaust and the X-line orientation are found to be $(0.93,0.32,-0.17)$ and $(0.10$, 0.24, 0.97), respectively in GSE coordinates. The intermediate to minimum eigenvalue ratio is about 5 (see Section 4 for further discussion). The normal magnetic field component is found to be close to zero but generally positive. The Alfvén speed $\left(\mathrm{V}_{\mathrm{A}}\right)$ is larger prior to the exhaust than after the exhaust (cf. values in Figure 3(f)). This asymmetry in $\mathrm{V}_{\mathrm{A}}$ leads to an asymmetry in the local magnetic shear at each exhaust boundary, as expected from the fact that one Alfvénic perturbation propagates slower than the other. This inferred geometry is sketched in Figure 1.

ACE, located somewhat closer to the Sun, observed the exhaust about 15 minutes earlier, as shown in Figure 4. The magnetic field and suprathermal electron data are quite similar to that observed at Wind, compatible with the crossing of the HCS, the encounter with a reconnection exhaust of similar spatial extent and duration, and the formation of closed field lines on the sunward side of a reconnection X-line. Within the exhaust, the electron pitch angle distribution (PAD) of panel (a) shows the mixing of the $0^{\circ}$ strahl from prior to the exhaust with the $180^{\circ}$ strahl from after the exhaust. This property is also observed within the exhaust at Wind, as shown in Figure 3(a). At both spacecraft, magnetic reconnection thus formed a region of closed field lines, with both ends connected to the Sun (Gosling et al., 2006). Figure 5 shows the ACE suprathermal electron PAD line curves for times just prior (blue), within (red) and after (black) the exhaust. The PAD from within the exhaust is clearly made up of a mixture of the strahl from both sides of the exhaust at $0^{\circ}$ and $180^{\circ}$ respectively (black and blue curves). MVA analysis gives similar results to those at Wind.

Figure 6 displays ST-A and ST-B suprathermal electron and magnetic field data around the times of their encounters with the HCS at 22:20 UT on March 31 and 01:25 UT on April 1, 2007, respectively. The magnetic field at ST-B shows a clear bifurcated signature, with the expected reversal of the magnetic field (panel (d)) and strahl (panel (c)) orientations across the HCS. This, the total magnetic shear of about $105^{\circ}$ across the HCS, and the temporal separation of 2 min between the bifurcated current sheets (though slightly longer than at ACE and Wind), makes this interval quite similar to the magnetic field measurements made at Wind and ACE. The suprathermal electron PAD plot clearly shows a mix of the strahl from both sides of the HCS within the bifurcated current sheets. Sample PAD curves, for times noted by arrows in panel (d), are shown in Figure 7(b) for various times at ST-B. Focusing for the time being on the solid lines only, we clearly observe counter-streaming suprathermal electrons within the bifurcated current sheets (red solid curve) which correspond to the mixing of the strahl from both sides of the HCS (black and blue curves). MVA results on ST-B magnetic field data gave a normal to the exhaust and Xline orientation vectors of $(0.79,0.26,-0.55)$ and $(0.44,0.38,0.81)$ in GSE coordinates, respectively. As observed in Figure 2, those orientations (blue dashed lines) are very close to those obtained at Wind (red dashed lines). The intermediate to minimum eigenvalue ratio at ST-B is above 10. Together with the results at ST-A (see later), the overall agreement between the orientations obtained at each spacecraft (cf. Figure 2) gives confidence in the results despite the relatively low eigenvalue ratios.

Although STEREO ion data (Galvin et al., 2008) do not permit an unambiguous identification of the magnetic reconnection-associated flow deflection (not shown), (1) the presence of bifurcated current sheets, (2) the clear mixing of the strahl from both sides of the HCS, (3) the close resemblance to the Wind and ACE magnetic field 
changes at the HCS (including the total magnetic shear), and (4) the very similar MVA results, strongly suggest that ST-B observed a magnetic reconnection exhaust that resulted from the same X-line as for Wind and ACE.

Observations at ST-A are overall similar, with a bifurcated current sheet observed around 22:20 UT, i.e., about the same time as the ACE exhaust encounter. The total magnetic shear across the HCS is about $95^{\circ}$, similar to those at the other spacecraft. The temporal extent of the reconnection exhaust is longer, however, about 8 min (Figure 6(c)). This corresponds to an exhaust 4 to 6 times larger spatially than at the other spacecraft, assuming simple planar exhaust geometry and the same background solar wind speed. Sample suprathermal electron PADs are shown in Figure 7(a) for ST-A. Focusing first on the solid lines, we see clear counter-streaming within the bifurcated current sheet, with a mixing of the strahl from both sides of the HCS. We take these signatures as strong evidence that ST-A also encountered a reconnection exhaust originating from the same X-line as the other spacecraft. MVA analysis yields a normal direction and an X-line orientation of $(0.72,0.57,-0.38)$ and $(0.21,0.36$, $0.91)$, respectively in GSE coordinates. The X-line orientation is similar to those at the other spacecraft in the Y$\mathrm{Z}_{\mathrm{GSE}}$ plane (dashed green line in Figure 2(a)). The exhaust intersection with the $\mathrm{X}-\mathrm{Y}_{\mathrm{GSE}}$ plane is, however, a bit different from those at the other spacecraft (dashed purple line in Figure 2(a)). The intermediate to minimum eigenvalue ratio is 5. At both ST-A and ST-B, the MVA gives a small but positive normal magnetic field component across the exhaust, compatible with the topology shown in Figure 1.

In addition to observing bifurcated currents and electron PAD signatures consistent with an exhaust and closed field lines rooted to the Sun, ST-A and ST-B observed counter-streaming suprathermal electrons outside the exhaust. Samples of such PADs are shown with dashed and dash-dotted lines in Figure 7(a) and 7(b), respectively for ST-A and ST-B, for times marked with arrows (with color correspondence) in Figures 6(b) and 6(d). These curves show the occurrence of strahl mixing, similar to that observed within the exhaust. However, these are sampled outside the exhaust, and on both sides of the main HCS at both STEREO spacecraft. Such structures outside the exhaust are interpreted as being within the electron separatrix layers. As depicted in Figure 1, owing to their large speed parallel to the magnetic field and to velocity dispersion effects, this separatrix layer is accessible to suprathermal electrons. The separatrix layer is supposedly directly adjacent to the exhaust, as illustrated in Figure 1. However, although both ST-A and ST-B observed such thin layers adjacent to the exhaust boundaries, they also observed the separatrix electrons in an intermittent fashion at substantial distances from the exhaust boundaries.

Interestingly, neither ACE nor Wind observed clear suprathermal electron separatrix layers (Figures 3(a) and 4(a)). The temporal resolution of suprathermal electron distributions at Wind (98 s) and ACE (64 s) are less than at both STEREO spacecraft (30 s). However, given the widths of the separatrix layers at both ST-A and ST-B this may not explain their absence at Wind and ACE. Their absence at Wind and ACE may be a result of the closeness of those spacecraft to the X-line. In such a case the separatrix layer has a finite and small spatial (and thus temporal) extent which might not be resolved by the instruments.

\section{Discussion and conclusions}

We have studied a solar wind magnetic reconnection exhaust event at the HCS observed by the Wind, ACE, STA and ST-B spacecraft between March 31 and April 1, 2007.

The sunward plasma flow deflection observed within the exhaust at Wind and ACE is consistent with all spacecraft being sunward of the magnetic reconnection X-line, at the time of observation, and with all four spacecraft being on closed magnetic field lines with both ends connected to the Sun. This is unambiguously demonstrated by the presence of counter-streaming strahls within the bifurcated current sheets at all spacecraft. The fact that plasma flow deflection is also strongly dawnward (Figure 3(d)) within the exhaust at Wind suggests that 
the magnetic reconnection $\mathrm{X}$-line at the origin of the exhaust is strongly tilted in the $\mathrm{Y}-\mathrm{Z}_{\mathrm{GSE}}$ plane. This is confirmed by the MVA analyses at all spacecraft. The X-line is thus located duskward of all spacecraft, as illustrated in Figure 2(b). The inferred X-line length for this event is likely at least $400-500 R_{E}$, i.e., from projection of the four spacecraft locations onto the dashed lines of Figure 2(b).

The distance from the inferred X-line to ST-A is very large. It is at least about $800 \mathrm{R}_{\mathrm{E}}$, i.e., the rough separation between ST-A and the ACE/Wind spacecraft along the direction perpendicular to the X-line in the Y-Z $\mathrm{Z}_{\mathrm{GSE}}$ plane (cf. Figure 2(b)). It is in fact likely more than $1100 \mathrm{R}_{\mathrm{E}}$, i.e., the actual distance between ACE and ST-A, which would be a minimum if the X-line was exactly along the GSE Z axis (since ACE and ST-A observe the exhaust at about the same time). This large distance from the X-line may explain the fact that the exhaust intersection with the $\mathrm{X}-\mathrm{Y}_{\mathrm{GSE}}$ plane deduced from MVA is not the same between Wind/ACE and ST-A (Figure 2(a)). Although errors in the MVA normal determinations are of the order of few degrees (from analytical and statistical bootstrap methods (Sonnerup and Scheible, 1998)), the exhaust and X-line orientations obtained at all spacecraft are similar and consistent with the dominant dawnward deflection at Wind, thus suggesting that these MVA orientations are overall correct. Assessment of the expected arrival times of the exhaust from the MVA orientations at each spacecraft gives different results depending on whether one uses that obtained at Wind or ST-A; each one leads to errors of the order of tens of minutes. This is in fact obvious from the geometry seen Figure 2(a). Since ACE and ST-A observe the exhaust at about the same time, actual timing assuming a planar exhaust would give an exhaust intersection with the X-Y plane corresponding to the red solid line, which orientation is just in between those obtained at Wind and ST-A (red and purple dashed lines in Figure 2(a)). The exhaust orientation in the X-Y $\mathrm{Y}_{\mathrm{GSE}}$ plane at ST-A is more tilted towards the Sun-Earth line than at Wind/ACE. This indicates a non-planar exhaust which was bent with the HCS, and in the sense of the Parker spiral orientation.

The large temporal extent of the exhaust at ST-A, $8 \mathrm{~min}$ as compared to about $1 \mathrm{~min} 20 \mathrm{~s}$ at Wind, is consistent with the fact that ST-A is more distant from the X-line. However, if we assume a uniform solar wind flow, a planar exhaust structure, and an exhaust wedge angle of $4^{\circ}$ (e.g., Gosling et al., 2007a), an exhaust observed as remote as $1000 \mathrm{R}_{\mathrm{E}}$ from the X-line should be about $225000 \mathrm{~km}$ thick $\left(\sim 35 \mathrm{R}_{\mathrm{E}}\right)$. This would correspond to $\sim 9$ min duration for a solar wind flow of $400 \mathrm{~km} / \mathrm{s}$ (as observed at Wind and in 10 min proton data at ST-A) and an exhaust perpendicular to the Sun-Earth line. Because the exhaust is very tilted towards the Sun-Earth line at ST-A, and because the X-line is likely located farther than $1000 \mathrm{R}_{\mathrm{E}}$, (since it ought to be duskward of Wind/ACE and even ST-B) the actual temporal extent of the exhaust should be significantly larger than 8 min. Furthermore, we believe that a wedge angle of $4^{\circ}$ can be viewed as a value on the low side of known reconnection rates. Thus, assuming an exhaust should linearly expand into the solar wind, the $8 \mathrm{~min}$ duration of the exhaust at ST-A would appear inconsistent with such a remote X-line location. But it is not known whether the wedge angle should persist at large distances from the X-line. Such a topic deserves further theoretical, simulation and statistical observational works, for which the large separations of the STEREO spacecraft will be useful.

We note, nevertheless, that (1) Wind and ACE observed an exhaust with very similar properties, (2) at the same HCS, (3) at about the same time, and (4) that the orientation of the X-line is almost along the GSE Z-axis at all spacecraft independently. These facts strongly suggest that it is the same exhaust that is seen at Wind/ACE and STA. The observations at ST-A are the result of magnetic reconnection when it was ongoing at the X-line likely more than 20 hours earlier, for an X-line $1000 \mathrm{R}_{\mathrm{E}}$ away and a propagation speed of $90 \mathrm{~km} / \mathrm{s}$ (cf. Alfvén speeds in Figure 3(f)). Thus, another possibility is that changes may have occurred at the X-line within this 20 -hours interval, e.g., in terms of either the location of the X-line or the reconnection rate. 
The inferred large distance between ST-A and the X-line also explains the presence of clear electron separatrix layers at ST-A while not at Wind and ACE, owing to simple geometric arguments.

The exhaust properties at ST-B are very similar to those at Wind and ACE (cf. Section 3). Still puzzling, however, are (1) the observation of clear electron separatrix layers at ST-B while not at Wind and ACE, and (2) the duration of the exhaust at ST-B, i.e., slightly longer than at Wind and ACE while it is inferred to be the closest to the X-line (Figure 2(b)) with similar MVA results. Both facts appear inconsistent with the scenario of stationary Xline and exhaust. The exhaust at ST-B is observed about 3 hours later than at ACE and ST-A, and thus it is likely that the reconnection process was subject to changes over this interval, either in terms of X-line location or reconnection rate. The fact that the electron separatrix layers are observed intermittently at ST-A and ST-B (Figure 6) is potentially also consistent with intermittent changes in X-line location or reconnection rate.

In conclusion, although at scales of the order of 400 to $700 \mathrm{R}_{\mathrm{E}}$ the reconnection process has been found to be surprisingly stationary in both space and time (e.g., Phan et al. 2006; Gosling et al. 2007a; 2007b), the present observations of a reconnection exhaust at very widely separated spacecraft across the same HCS (over $1800 \mathrm{R}_{\mathrm{E}}$ between ST-A and ST-B) highlights the fact that the reconnection process may become non-stationary, and certainly more complex, at such very large spatial and temporal scales in the solar wind.

Finally, we emphasize that counter-streaming suprathermal electrons have been commonly found in the vicinity of the HCS (Pilipp et al., 1987). From a large statistical analysis of suprathermal electron data, Feuerstein et al. (2004) showed that counter-streaming electron fluxes are nearly always unbalanced. Their dataset showed no clear separate population of balanced counter-streaming fluxes that would represent times of closed loops such as magnetic clouds. Such a general feature may have two main interpretations. Intersection with the leg of a closed loop extending much farther out, as opposed to sampling near its apex, may result in higher anti-sunward than sunward fluxes because the sunward-directed electrons travelled further and thus were subject to scattering for a longer time (cf. Pilipp et al., 1987; Kahler, Crooker, and Gosling, 1999a; 1999b). A second possibility is that the different intensities owe to different source populations at the loop roots on the Sun (e.g., Gosling et al., 2006). In a similar fashion to the case of Gosling et al. (2006), the present event provides evidence for the latter. The suprathermal electron PADs within the exhausts and adjacent separatrix layers are marked by strong asymmetry between the field-aligned and anti-field-aligned phase space density (PSD) values. Closed magnetic field lines resulting from reconnection in the solar wind need not have balanced counter-streaming electron PSD, even though in such a case the spacecraft samples near the apex of the newly closed flux tubes: the imbalance thus merely results from different strahl source populations. This provides further evidence that intervals of closed solar wind field lines can be, and likely have been, misidentified if the strahls strengths from the two opposite ends of the field lines were significantly different. This is consistent with the observation of Feuerstein et al. (2004) that there is statistically no distinct population of balanced counter-streaming electron fluxes. In addition to the known suprathermal electron counter-streaming patterns due to shocks (Gosling et al., 1993; Steinberg et al., 2005) and halo depletions at $90^{\circ}$ pitch angle (Gosling, Skoug, and Feldman, 2001; Skoug et al., 2006), this renders identification of closed heliospheric magnetic field somewhat complicated. 
Acknowledgments. The authors are grateful to the STEREO, ACE and Wind instrument teams and the CDAWeb for providing part of the data. C. F. acknowledges financial support from the UK Science and Technology Facilities Council (STFC) on the MSSL Rolling Grant.

\section{References}

Acuña, M. H., Curtis, D., Scheifele, J. L., Russell, C. T., Schroeder, P., Szabo, A., Luhmann, J. G.: 2008, Space Sci. Rev. 136, 203.

Davis, M. S., Phan, T. D., Gosling, J. T., Skoug, R. M.: 2006, Geophys. Res. Lett. 33, 19102, doi:10.1029/2006GL026735.

Dungey, J. W.: 1961, Phys. Rev. Lett. 6, 47.

Feuerstein, W. M., Larson, D. E., Luhmann, J. G., Lin, R. P., Kahler, S. W., Crooker, N. U.: 2004, Geophys. Res. Lett. 31, 22805, doi:10.1029/2004GL020529.

Fuselier, S. A., Anderson, B. J., Onsager, T. G.: 1995, J. Geophys. Res. 100, 11,805.

Fuselier, S. A., Anderson, B. J., Onsager, T. G.: 1997, J. Geophys. Res. 102, 4847.

Galvin, A. B., Kistler, L. M., Popecki, M., Farrugia, C. J., Simunac, K. D. C., Ellis, L., Möbius, E., Lee, M. A., Boehm, M., Carroll, J., et al.: 2008, Space Sci. Rev. 136, 437.

Gosling, J. T., Thomsen, M. F., Bame, S. J., Russell, C. T.: 1986, J. Geophys. Res. 91, 356.

Gosling, J. T., Thomsen, M. F., Bame, S. J., Onsager, T. G., Russell, C. T.: 1990, Geophys. Res. Lett. 17, 1833.

Gosling, J. T., Bame, S. J., Feldman, W. C., McComas, D. J., Phillips, J. L., Goldstein, B. E.: 1993, Geophys. Res. Lett. $20,2335$.

Gosling, J. T., Skoug, R. M., Feldman, W. C.: 2001, Geophys. Res. Lett. 28, 4155.

Gosling, J. T., Skoug, R. M., McComas, D. J., Smith, C. W.: 2005a, J. Geophys. Res. 110, A01107, doi:10.1029/2004JA010809

Gosling, J. T., Skoug, R. M., McComas, D. J., Smith, C. W.: 2005b, Geophys. Res. Lett. 32, 05105, doi:10.1029/2005GL022406.

Gosling, J. T., McComas, D. J., Skoug, R. M., Smith, C. W.: 2006, Geophys. Res. Lett. 33, 17102, doi:10.1029/2006GL027188.

Gosling, J. T., Eriksson, S., Phan, T. D., Larson, D. E., Skoug, R. M., McComas, D. J.: 2007a, Geophys. Res. Lett. 34, 06102, doi10.1029/2006GL029033.

Gosling, J. T., Phan, T. D., Lin, R. P., Szabo, A.: 2007b, Geophys. Res. Lett. 34, 15110, doi10.1029/2007GL030706.

Gosling, J. T., Eriksson, S., Blush, L. M., Phan, T. D., Luhmann, J. G., McComas, D. J., Skoug, R. M., Acuna, M. H., Russell, C. T., Simunac, K. D.: 2007c, Geophys. Res. Lett. 34, 20108, doi:10.1029/2007GL031492.

Huttunen, K. E. J., Bale, S. D., Salem, C.: 2008, Ann. Geophys. 26, 2701.

Kahler, S. W., Crooker, N. U., Gosling, J. T.: 1999a, J. Geophys. Res. 104, 9911.

Kahler, S. W., Crooker, N. U., Gosling, J. T.: 1999b, J. Geophys. Res. 104, 9919.

Kaiser, M. L., Kucera, T. A., Davila, J. M., St. Cyr, O. C., Guhathakurta, M., Christian, E.: 2008, Space Sci. Rev. $136,5$.

Lavraud, B., Thomsen, M. F., Taylor, M. G. G. T., Wang, Y. L., Phan, T. D., Schwartz, S. J., Elphic, R. C., Fazakerley, A., Rème, H., Balogh, A.: 2005, J. Geophys. Res. 110, A06209, doi:10.1029/2004JA010808.

Lavraud, B., Thomsen, M. F., Lefebvre, B., Schwartz, S. J., Seki, K., Phan, T. D., Wang, Y. L., Fazakerley, A., Rème, H., Balogh, A.: 2006, J. Geophys. Res. 111, A05211, doi:10.1029/2005JA011266.

Lepping, R. P., Acũna, M. H., Burlaga, L. F., Farrell, W. M., Slavin, J. A., Schatten, K. H., Mariani, F., Ness, N. F., Neubauer, F. M., Whang, Y. C., et al.: 1995, Space Sci. Rev. 71, 207.

Lin, R. P., Anderson, K. A., Ashford, S., Carlson, C., Curtis, D., Ergun, R., Larson, D., McFadden, J., McCarthy, M., Parks, G. K., et al.: 1995, Space Sci. Rev. 71, 125

Luhmann, J. G., Curtis, D. W., Schroeder, P., McCauley, J., Lin, R. P., Larson, D. E., Bale, S. D., Sauvaud, J.-A., Aoustin, C., Mewaldt, R. A., et al.: 2008, Space Sci. Rev. 136, 117.

McComas, D. J., Bame, S. J., Barker, P., Feldman, W. C., Phillips, J. L., Riley, P., Griffee, J. W.: 1998, Space Sci. Rev. 86, 563.

Onsager, T. G., Scudder, J. D., Lockwood, M., Russell, C. T.: 2001, J. Geophys. Res. 106, 25,467.

Paschmann, G., Sonnerup, B. U. Ö., Papamastorakis, I., Sckopke, N., Haerendel, G.: 1979, Nature 282, 243.

Paschmann, G., Baumjohann, W., Sckopke, N., Phan, T. D., Luehr, H.: 1993, J. Geophys. Res. 98, 13,409.

Phan, T. D., Gosling, J. T., Davis, M. S., Skoug, R. M., Øieroset, M., Lin, R. P., Lepping, R. P., McComas, D. J., Smith, C. W., Reme, H., Balogh, A.: 2006, Nature 439, 175.

Pilipp, W. G., Muehlhaeuser, K.-H., Miggenrieder, H., Rosenbauer, H., Schwenn, R.: 1987, J. Geophys. Res. $92,1103$.

Russell, C. T., Elphic, R. C.: 1978, Space Sci. Rev. 22, 681.

Russell, C. T., Elphic, R. C.: 1979, Geophys. Res. Lett. 6, 33.

Sauvaud, J.-A., Larson, D., Aoustin, C., Curtis, D., Médale, J.-L., Fedorov, A., Rouzaud, J., Luhmann, J., Moreau, T., Schröder, P., et al.: 2008, Space Sci. Rev. 136, 227.

Skoug, R. M., Gosling, J. T., McComas, D. J., Smith, C. W., Hu, Q.: 2006, J. Geophys. Res. 111, A01101, doi:10.1029/2005JA011316.

Smith, C. W., L'Heureux, J., Ness, N. F., Acuña, M. H., Burlaga, L. F., Scheifele, J.: 1998, Space Sci. Rev. 86, 613.

Sonnerup, B. U. O., Paschmann, G., Papamastorakis, I., Sckopke, N., Haerendel, G., Bame, S., Asbridge, J., Gosling, J., Russell, C.: 1981, J. Geophys. Res. 86, 10,049.

Sonnerup, B. U. O., Scheible, M.: 1998, Int. Space Sci. Inst. Scientific Report Book 185.

Steinberg, J. T., Gosling, J. T., Skoug, R. M., Wiens, R. C.: 2005, J. Geophys. Res. 110, A06103, doi:10.1029/2005JA011027. 


\section{FIGURES:}

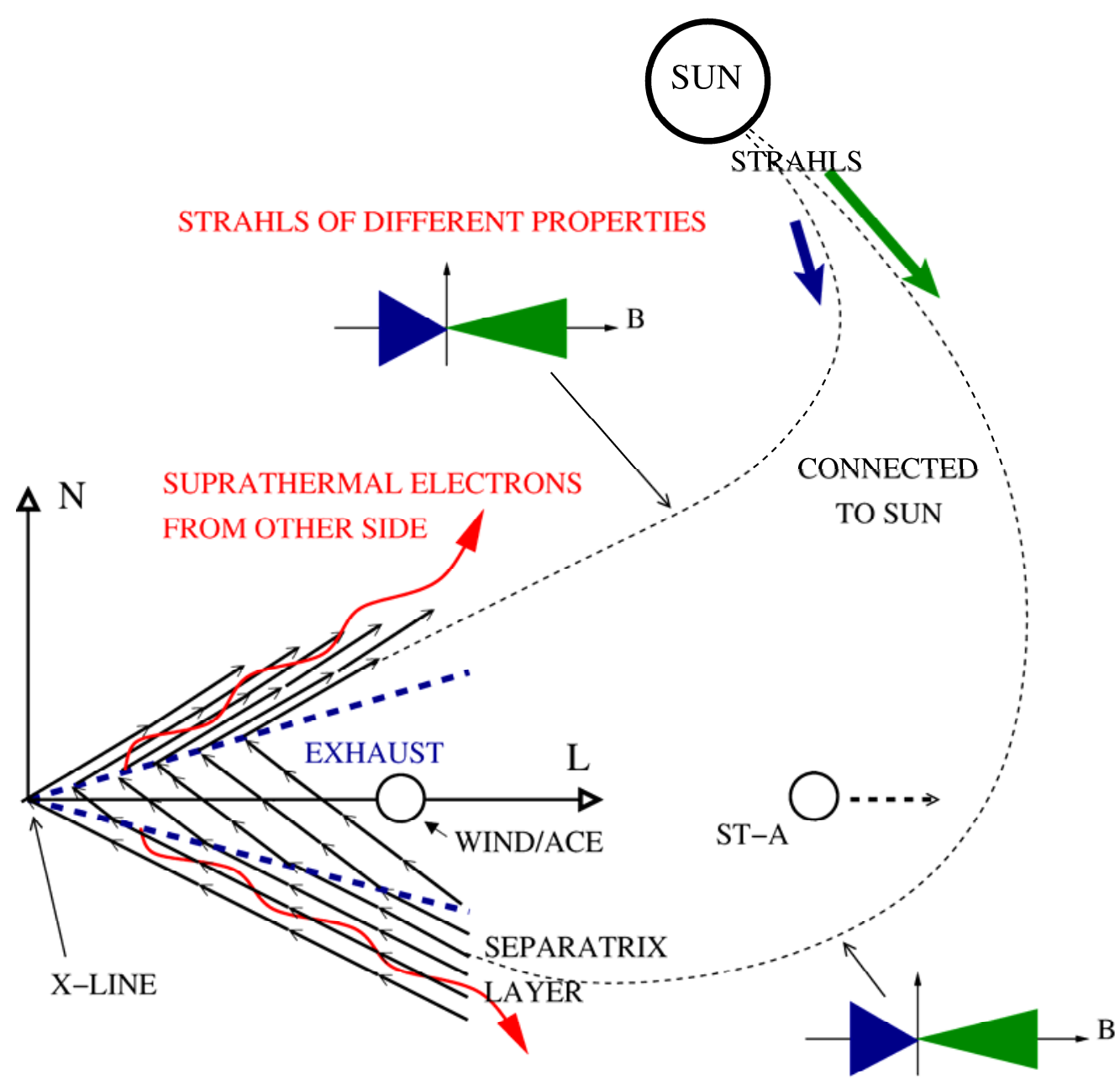

Figure 1. (Lower left part) Highly idealized planar projection of an asymmetric reconnection exhaust convecting with the solar wind (grossly as deduced from MVA analysis and in the L-N plane). The sharp field line kink produced by reconnection propagates as a pair of Alfvénic disturbances parallel and antiparallel to a reconnected field line into the plasma on opposite sides of the reconnecting current sheet. As the Alfvénic disturbances propagate they steepen into a pair of back-to-back rotational-like discontinuities (dashed blue lines) that bound the exhaust. These accelerate the plasmas they intercept into the exhaust and away from the reconnection site. The exhaust boundaries are rotational-like discontinuities and are thus locally open. Suprathermal electrons can flow from one side of the exhaust to the other, as illustrated with curvy red lines, so as to form electron separatrix layers. (Right part of figure) Spacecraft such as Wind/ACE and ST-A, located on the sunward side of the X-line are on newly closed field lines and thus observe the mixing of the suprathermal electron strahls from the two sides of the reconnecting heliospheric current sheet. The strahls from the two sides are in this case of different strengths (blue and green arrows at the Sun), and form asymmetric, counter-streaming populations in pitch angle distributions, such as those illustrated, in both the exhaust and the separatrix layers. Adapted from Gosling et al. (2005a). 


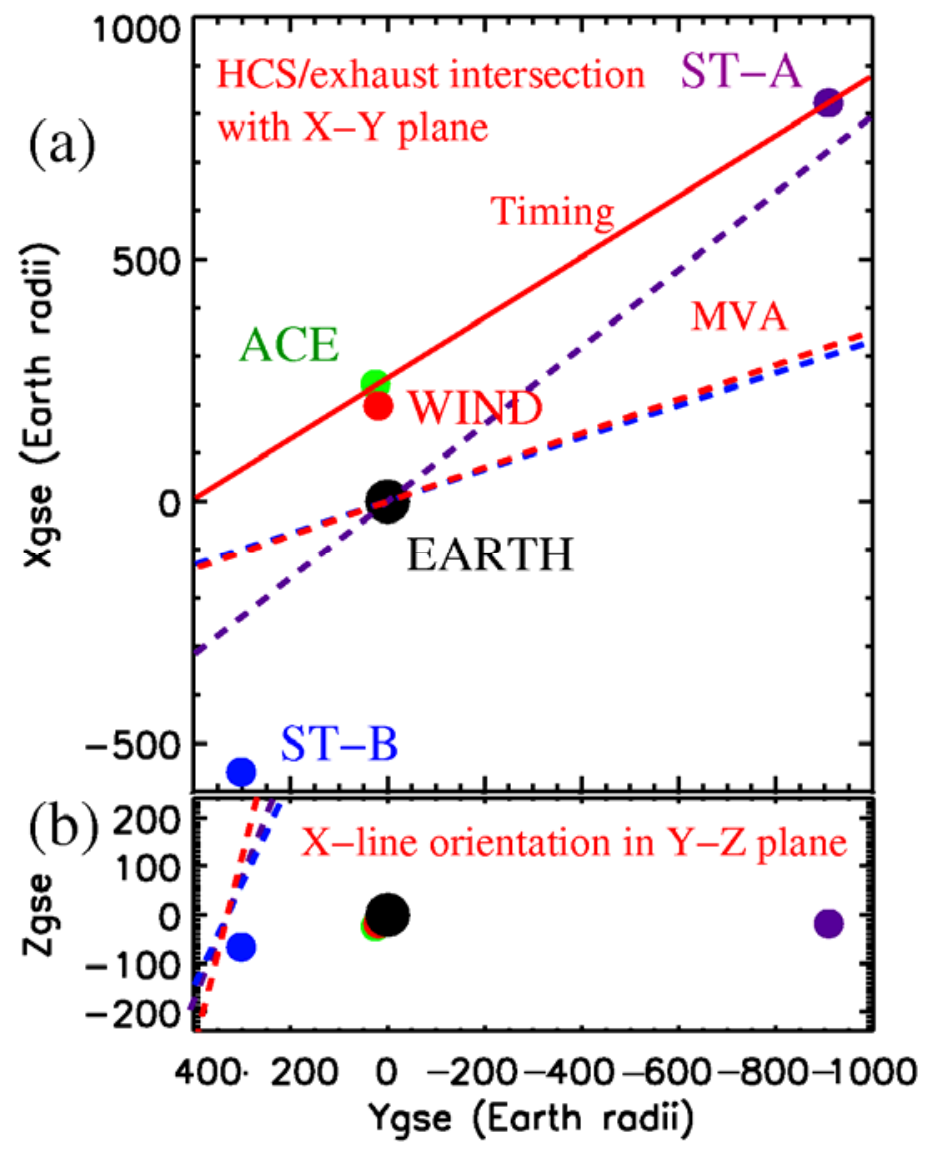

Figure 2. Spacecraft locations in GSE coordinates at 22:30 UT on 31 March, 2007. (a) The exhaust intersection with the $\mathrm{X}-\mathrm{Y}$ plane as calculated from timing between ACE and ST-A is shown as a red solid line. Those obtained from MVA analyses at Wind, ST-A and ST-B are displayed with red, purple and blue dashed lines, respectively. (b) The X-line orientations in the Y-Z plane, as deduced from MVA analyses at each spacecraft, are shown with the same color coding. 


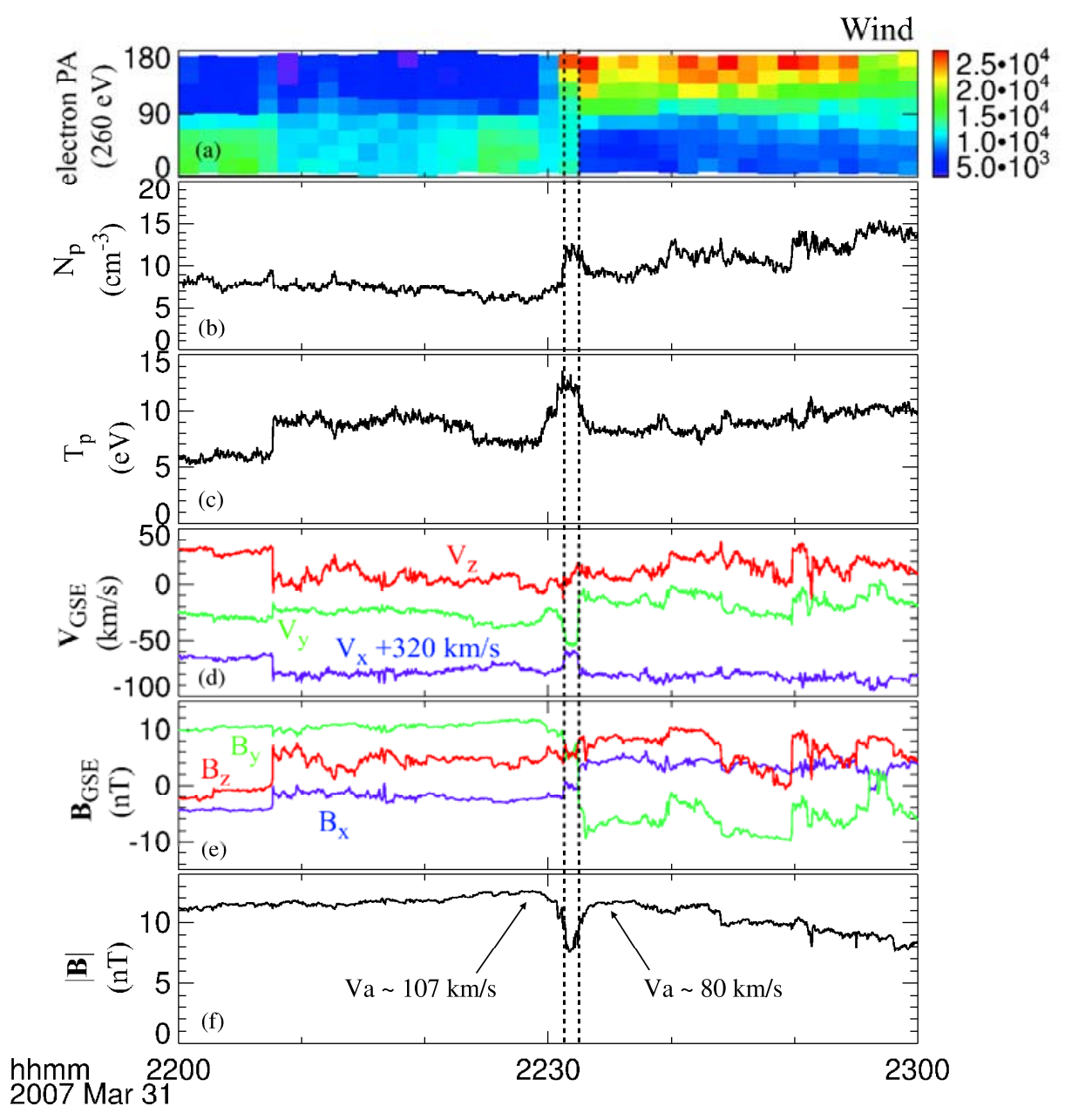

Figure 3. WIND data for the interval around the solar wind reconnection exhaust (marked with vertical dashed lines) observed at $\sim 22: 32$ UT on 31 March, 2007. Panel (a) shows a $\sim 260 \mathrm{eV}$ suprathermal electron pitch angle spectrogram. Panels (b), (c) and (d) show the proton density, temperature and velocity GSE components, respectively. Panels (e) and (f) show the magnetic field GSE components and its magnitude, respectively. Alfvén speeds on either side of the exhausts are given in panel (f). 


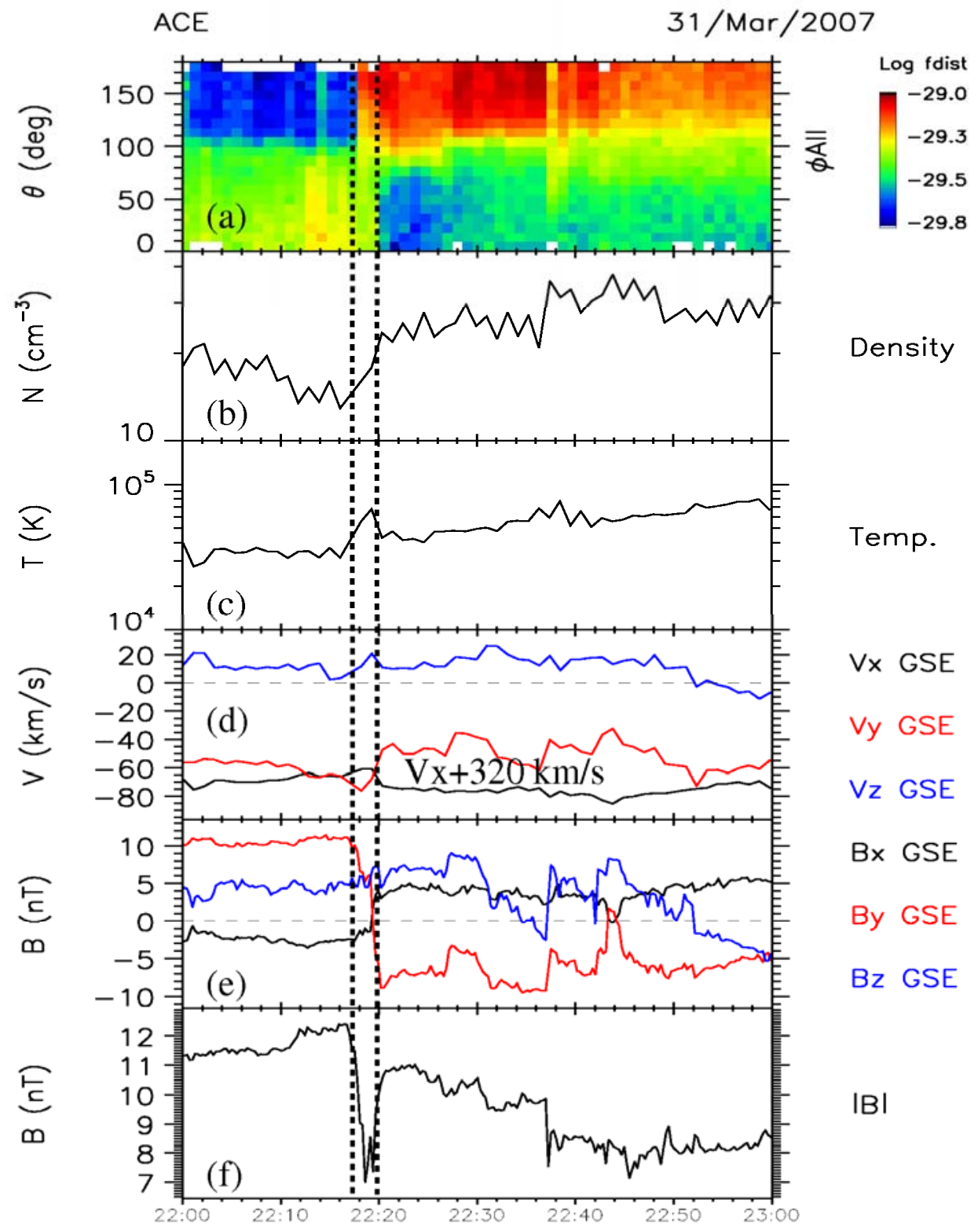

Figure 4. ACE data for the interval around the solar wind reconnection exhaust (marked with vertical dashed lines) observed at 22:20 UT on 31 March, 2007. Panel (a) shows a $\sim 272 \mathrm{eV}$ suprathermal electron pitch angle spectrogram. Panels (b), (c) and (d) show the proton density, temperature and velocity GSE components, respectively. Panels (e) and (f) show the magnetic field GSE components and its magnitude, respectively. 


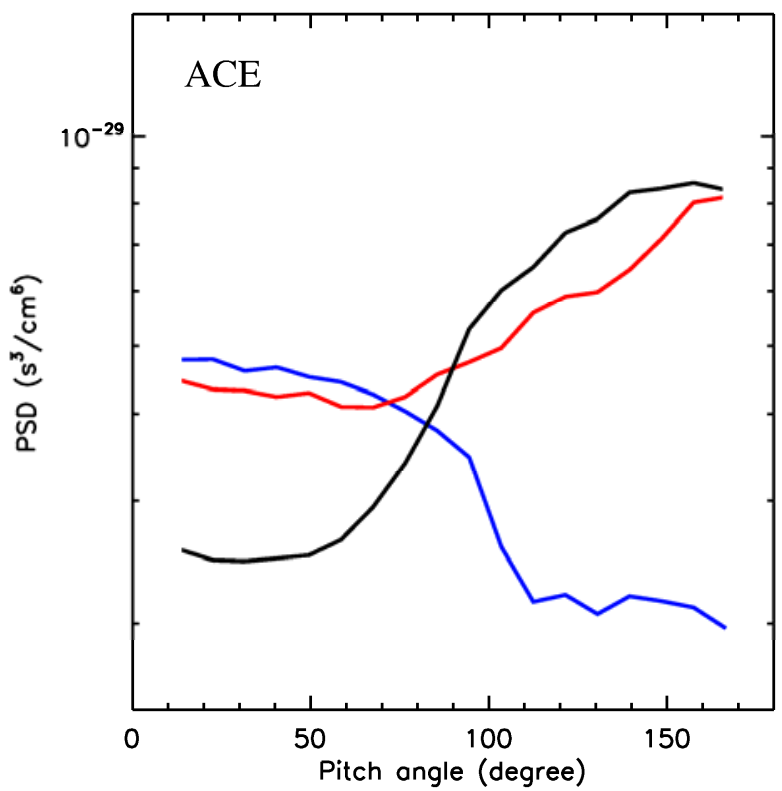

Figure 5. ACE $272 \mathrm{eV}$ suprathermal electron pitch angle distributions obtained just before (blue), within (red) and after (black) the exhaust. Each is an average of two consecutive PAD samples. 

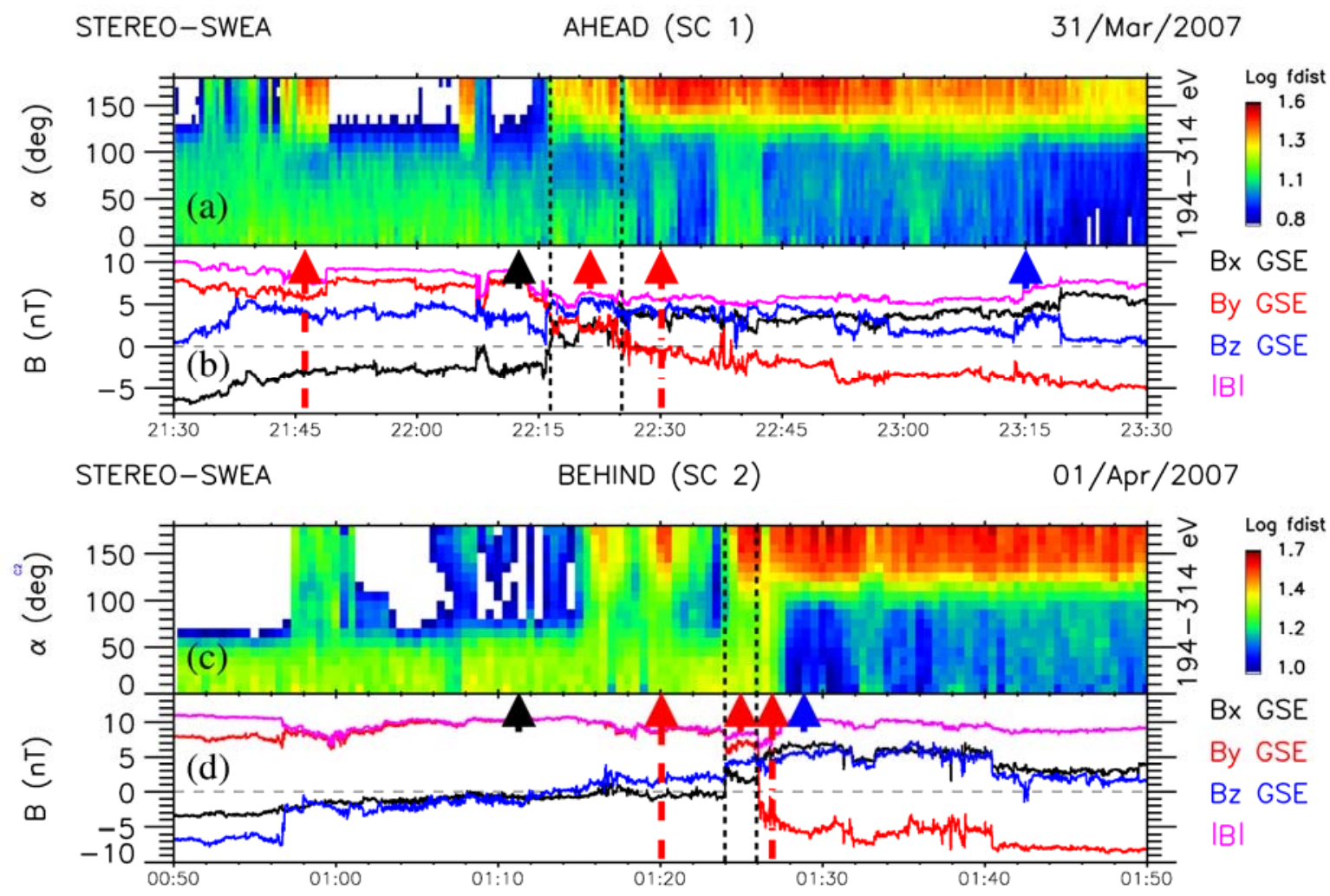

Figure 6. ST-A (panels (a) and (b)) and ST-B (panels (c) and (d)) data for the intervals around the solar wind reconnection exhausts encountered at each spacecraft (marked with vertical dashed lines). Panels (a) and (c) show a $\sim 250$ eV suprathermal electron PAD spectrogram. Panels (b) and (d) show the magnetic field magnitude and GSE components. Arrows in panels (b) and (d) show the times of the suprathermal electron PADs displayed in Figure 7, with the corresponding line style and color coding. 

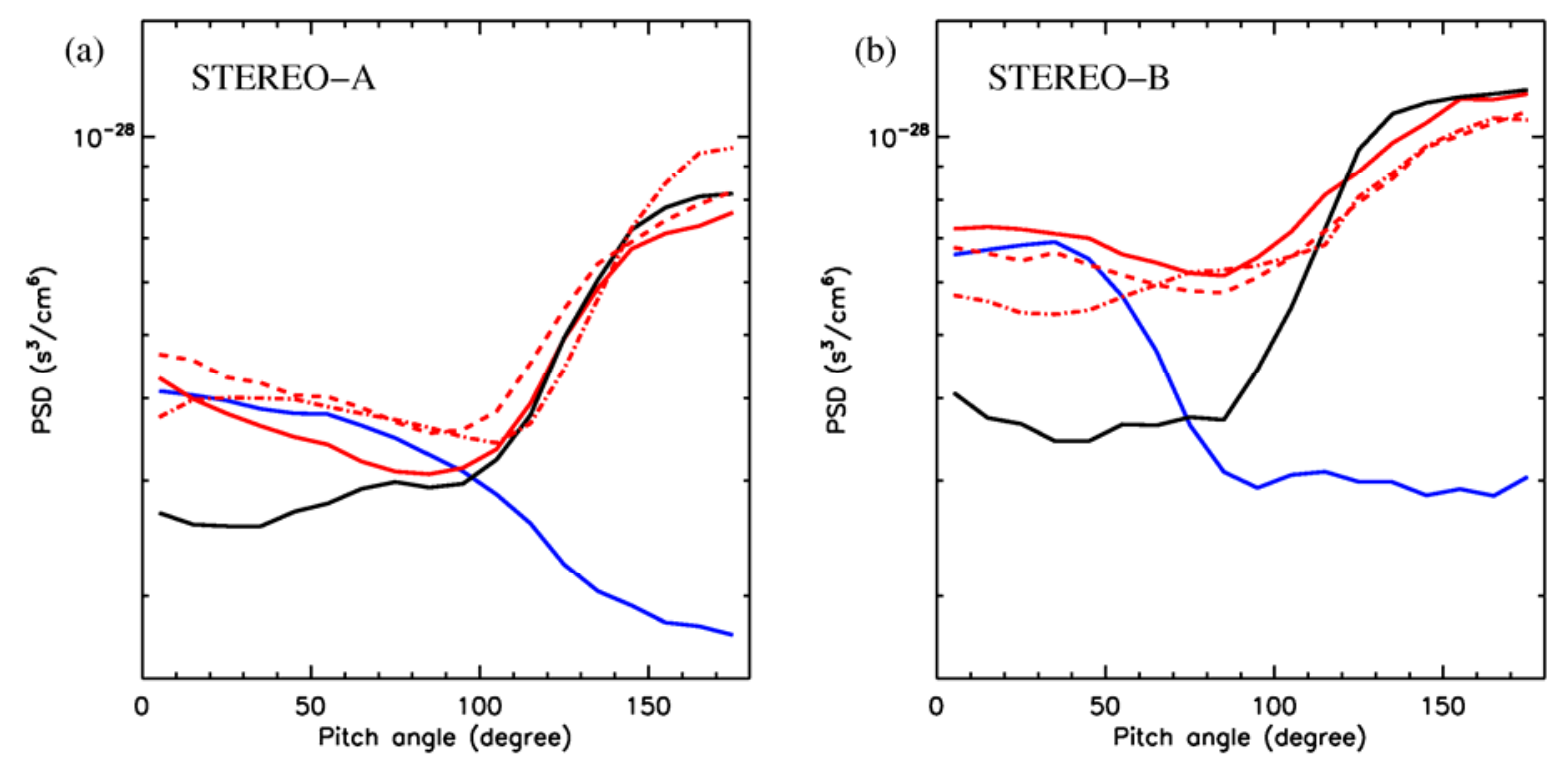

Figure 7. ST-A (a) and ST-B (b) $\sim 250 \mathrm{eV}$ suprathermal electron pitch angle distributions obtained just before (blue), within (red) and after (black) the exhausts observed at each spacecraft. Each is an average of three consecutive PAD samples. 